Journal for ImmunoTherapy of Cancer

\section{short-course enzalutamide alone and with immunotherapy in non-metastatic castration sensitive prostate cancer}

To cite: Madan RA, Karzai F, Donahue RN, et al. Clinical and immunologic impact of shortcourse enzalutamide alone and with immunotherapy in nonmetastatic castration sensitive prostate cancer. Journal for ImmunoTherapy of Cancer 2021;9:e001556. doi:10.1136/ jitc-2020-001556

- Additional material is published online only. To view please visit the journal online (http://dx.doi.org/10.1136/jitc2020-001556)

Accepted 03 November 2020

Check for updates

(C) Author(s) (or their employer(s)) 2021. Re-use permitted under CC BY-NC. No commercial re-use. See rights and permissions. Published by BMJ.

${ }^{1}$ Genitourinary Malignancies, Center for Cancer Research, National Cancer Institute, Bethesda, Maryland, USA ${ }^{2}$ Laboratory of Tumor Immunology and Biology, Center for Cancer Research, National Cancer Institute, Bethesda, Maryland, USA

${ }^{3}$ The Center for Prostate Disease Research, Walter Reed National Military Medical Center, Bethesda, Maryland, USA

Correspondence to

Dr Ravi A Madan;

madanr@mail.nih.gov

\section{ABSTRACT}

Background The standard treatment for non-metastatic castration sensitive prostate cancer (nmCSPC) is androgen deprivation therapy (ADT) or surveillance. This study evaluated the potential synergy of immunotherapy and enzalutamide (without ADT) in nmCSPC. In addition, the immunologic impact of enzalutamide was also evaluated in men with normal testosterone.

Methods Patients with rising prostate-specific antigen (PSA) after definitive therapy, normal testosterone and no radiographic metastasis were randomized to enzalutamide for 3 months with/without PROSTVAC for 6 months. Thereafter, patients could be retreated with another 3 month course of enzalutamide when PSA returned to baseline. Immune profiles were evaluated in these patients.

Results Thirty-eight patients were randomized with a median $\mathrm{PSA}=4.38 \mathrm{ng} / \mathrm{dL}$ and $\mathrm{PSA}$ doubling time $=4.1$ months. No difference was observed between the two groups for PSA growth kinetics, but PSA responses to enzalutamide were noteworthy regardless of PROSTVAC. The median PSA decline after short-course enzalutamide without ADT/testosterone lowering therapy was $99 \%$ in both courses. The median time to PSA recovery to baseline after each 84-day course of enzalutamide was also noteworthy because of the duration of response after enzalutamide was discontinued. After the first and second 3 month cycle of enzalutamide, PSA recovery to baseline took a median 224 (range 84-1246) and 189 days (78-400), respectively. The most common adverse events related to the enzalutamide were grade 1 fatigue $(71 \%)$ and grade 1 breast pain/nipple tenderness (81\%). The only grade 3 toxicity was aspartate aminotransferase (AST)/alanine aminotransferase (ALT) elevation in two patients. Enzalutamide was independently associated with immune changes, increasing natural killer cells, naïve-T cells, and decreasing myeloid-derived suppressor cells. Conclusions Three months of enzalutamide without ADT induced substantial PSA control beyond the treatment period and was repeatable, perhaps representing an alternative to intermittent ADT in nmCSPC. In addition, enzalutamide was associated with immune changes that could be relevant as future immune combinations are developed.

Trail registration number clinicaltrials.gov (NCT01875250).

\section{BACKGROUND}

Enzalutamide is an androgen receptor inhibitor that initially demonstrated efficacy in patients with metastatic castration resistant prostate cancer (mCRPC). ${ }^{12}$ In recent years, trials in earlier stages of prostate cancer including metastatic castration sensitive prostate cancer (mCSPC) and non-mCSPC (nmCSPC) have demonstrated the clinical benefit of using enzalutamide before the development of mCRPC. ${ }^{34}$ Another subpopulation worthy of investigation are those with a rising prostate-specific antigen (PSA) after definitive surgery or radiation, but without evidence of metastatic disease on conventional imaging (CT/MRI and Tc99 bone scan).

Approximately 25000 men in the USA develop nmCSPC annually, also commonly referred to as biochemically recurrent prostate cancer. Standard treatment includes observation until metastatic disease or androgen-deprivation therapy (ADT) based on patient preference or PSA doubling time (PSA DT) ${ }^{5-7}$ Retrospective data suggest that PSA DT can be used to identify patients who are at highest risk for metastatic disease. ${ }^{89}$ There is also retrospective data suggesting that altering the PSA DT/PSA velocity could impact survival in nmCSPC, but prospective trials are required. ${ }^{10}$ 
A single previous study that is published explored the use of indefinite enzalutamide without ADT in nmCSPC and presented efficacy data and toxicities after 25 weeks. ${ }^{11}$ While these findings are important, efforts should be made to develop strategies to minimize toxicity in an asymptomatic population like nmCSPC. Another trial has demonstrated that intermittent $\mathrm{ADT}$ is equivalent to continuous ADT in nmCSPC. ${ }^{12}$

Based on that data and preclinical data suggesting the potential synergy of enzalutamide with immunotherapy, we conducted a randomized trial of a 3-month course of enzalutamide with and without PROSTVAC, a poxviral based therapeutic cancer vaccine targeting PSA. ${ }^{13} 14$ Although PROSTVAC was not effective as a single agent to extend survival in mCRPC, data suggest that it can be synergistic with enzalutamide and perhaps slow tumor growth rates, which would be especially important in nmCSPC. ${ }^{14-16}$ Immune monitoring was also conducted to evaluate the immunologic impact of enzalutamide and the combination of enzalutamide and PROSTVAC in this population.

\section{METHODS}

This was a single institution trial conducted at the National Cancer Institute, NIH, Bethesda, Maryland, USA. Patients enrolled from July 2013 to June 2015 (online supplemental figure 1).

Eligible patients were required to have had previous definitive therapy with either radiation or surgery. Patients were required to have negative CT scans/MRI and Tc99 bone scans. The eligible PSA range was between 2.0 and $20.0 \mathrm{ng} / \mathrm{mL}$ with a PSA DT of less than 12 months. Because of the use of PROSTVAC, patients with autoimmune diseases and requirements for chronic steroids were excluded. Patients who had previous chemotherapy were also excepted. Herbal medications and other medications known to influence PSA were prohibited. Previous ADT was allowed in patients with a normal range testosterone at study entry.

All patients were treated with enzalutamide $160 \mathrm{mg}$ orally (in the absence of ADT) for 84 days. Patients randomized to the vaccine arm were also given PROSTVAC-V (vaccinia, $2 \times 10^{8}$ infectious units subcutaneous) at week 1 , followed by PROSTVAC-F (fowlpox $1 \times 10^{9}$ infectious units) at weeks $3,5,9,13,17$ and 21. Per protocol, patients were then followed every 4-6 weeks to track PSA recovery to their respective baseline value. After an amendment to the study protocol, patients could have a second 84-day course of enzalutamide if the PSA recovery to baseline was beyond 7 months from treatment initiation and the CT and Tc99 bone scan remained negative for metastatic disease. (No further vaccine was administered.) Thereafter patients were followed until PSA recovery to baseline.

\section{Analysis of antigen-specific T cell responses, peripheral immune subsets, and thymic activity}

Antigen-specific $\mathrm{T}$ cell responses against PSA were analyzed in cryopreserved peripheral blood mononuclear cells (PBMCs) collected from patients before and during therapy, by intracellular cytokine staining following a period of in vitro stimulation with overlapping peptides pools using methods previously described. ${ }^{17}$ The PSA peptide pools contained a previously identified agonist epitope, and peptide pools encoding human leukocyte antigen and a mixture of peptides of cytomegalovirus, Epstein-Barr virus, influenza, and tetanus toxin served as negative and positive controls, respectively. ${ }^{18}$

Cryopreserved PBMCs collected from patients before and during therapy were examined by multicolor flow cytometry to identify 123 peripheral immune cell subsets, using the methodology previously described. ${ }^{19}{ }^{20}$ Subsets evaluated included nine parental cell types (CD4+ and CD8+ T cells, T-regulatory cells, natural killer (NK) cells, NK-T cells, conventional dendritic cells, plasmacytoid dendritic cells, B cells, and myeloid-derived suppressor cells (MDSCs)) and 114 refined subsets related to the maturation/function of the parental cell types. Flow cytometry files were analyzed using FlowJo V9.7 for Macintosh using the outlined gating strategy (online supplemental figure 2) with non-viable cells excluded and negative gates based on fluorescence minus 1 controls. The frequency of all subsets was calculated as a percentage of PBMC to help eliminate the bias that could occur in the smaller populations with fluctuations in leukocyte subpopulations. Immune subsets with a potentially biologically relevant change following therapy were defined as those with $\mathrm{p}<0.05$, the majority of patients having a $>25 \%$ change, difference in medians $>0.05 \%$ of PBMCs, and a frequency $>0.01 \%$ of total PBMCs. Multiple prior studies have used this method to detect the dynamics of 123 immune subsets, many of which have known biologic functions. ${ }^{21-24}$

T cell receptor excision circles (TRECs), a byproduct formed during $\mathrm{T}$ cell receptor rearrangement, which identifies recent thymic emigrants, was analyzed on DNA extracted from PBMC, using methods previously described. ${ }^{25} 26$

Impact of enzalutamide on vascular endothelial growth factor Preliminary data have suggested that enzalutamide could potentially impact vascular endothelial growth factor (VEGF) and this study involving enzalutamide without ADT provided an opportunity to evaluate that, independent of the immune analysis. ${ }^{27}{ }^{28}$ Venous blood was obtained by uncomplicated venipuncture into an EDTA tube at baseline, 4 weeks, 12 weeks and pre and post second course of enzalutamide. Samples were immediately placed on ice and processed within 2 hours of collection. Blood was centrifuged for $5 \mathrm{~min}$ at $1200 \mathrm{~g}$ and plasma stored at -80 Celsius until time of analysis. VEGF concentrations were measured by a commercial ELISA assay (R\&D Systems Quantikine Human VEGF Immunoassay), following manufacturer's instructions. All samples were evaluated in duplicate and an internal control was included in each plate. The lower limit of quantitation of the assay was $9.0 \mathrm{pg} / \mathrm{mL}$. 
Table 1 Baseline characteristics

\begin{tabular}{llll}
\hline & Enzalutamide+PROSTVAC & Enzalutamide & All Patients \\
\hline Number of Patients & 19 & 19 & 38 \\
Median Age (Range) & 64.4 years (56-79) & 68 years (54-87) & $64.6(54-87)$ \\
Gleason & & & 6 \\
\hline 6 & 1 & 5 & 15 \\
7 & 9 & 6 & 17 \\
$8-10$ & 9 & 8 & $4.38 \mathrm{ng} / \mathrm{dL}(2.02-19.43)$ \\
\hline Median PSA (Range) & $3.94 \mathrm{ng} / \mathrm{dL}(2.02-19.43)$ & $4.49 \mathrm{ng} / \mathrm{dL}(2.25-16.75)$ & $4.1 \mathrm{months}(0.9-10.3)$ \\
Median PSA Doubling Time (Range) & $3.3 \mathrm{months}(0.9-7.8)$ & $5.2 \mathrm{months} \mathrm{(1.6-10.3)}$ & $316 \mathrm{ng} / \mathrm{dL}(167-723)$ \\
Median Testosterone (Range) & $314 \mathrm{ng} / \mathrm{dL}(182-472)$ & $317 \mathrm{ng} / \mathrm{dL}(167-723)$ & $14(37 \%)$ \\
\hline Previous ADT (Percent) & $7(37 \%)$ & 7 (37\%) & \\
\hline
\end{tabular}

\section{Statistical design}

The study was designed to evaluate if PROSTVAC could slow the PSA growth rate after a short-course of enzalutamide. The primary endpoint of the trial was to compare the PSA recovery kinetics at 7 months after enzalutamide initiation between the two arms of the study based on previous data suggesting that vaccines could slow tumor growth rate over time. ${ }^{29} 30$ This was based on retrospective data that suggested vaccines may slow the growth rates in prostate cancer as measured by PSA. With 17 patients in each arm, there would be $80 \%$ power to detect a 1 SD difference between the two groups with a two-tailed 0.05 significance level two-group t-test. Up to 38 total patients were allowed to be enrolled, in case a small number of patients were found to be inevaluable. The growth rate was measured via the equation $f(t)=\exp (-d *$ $t)+\exp (g * t)-1$, where $\exp$ is the base of the natural logarithm, $e=2.7182$ and $\mathrm{t}$ is days since treatment initiation,

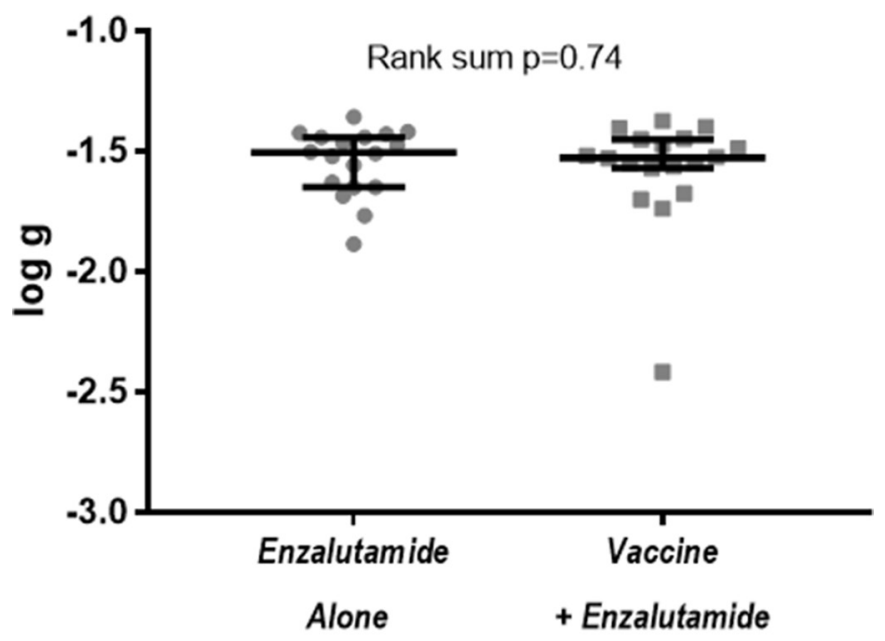

\section{Median log g with $95 \% \mathrm{Cl}$}

Figure 1 PSA Growth Rates. There was no difference seen in PSA growth rates in the 4 months following enzalutamide discontinuation regardless of the 6-month course of PROSTVAC administered to the combination group. Growth rates defined as previously described. ${ }^{16}$ as previously described. ${ }^{16}$ Secondary objectives included safety, impact of short-course enzalutamide on PSA kinetics, changes in testosterone, and VEGF levels.

\section{RESULTS}

The two groups $(n=38)$ were relatively balanced at enrollment (table 1). The growth rate as measured via the prespecified equation was not different between the two groups (0.031 for enzalutamide alone vs 0.030 for the combination; $\mathrm{p}=0.74$ by Wilcoxon rank sum test) after 7 months (figure 1).

Although the trial's primary endpoint was not met, the impact of the short-course of enzalutamide in all patients, regardless of PROSTVAC, is noteworthy. Thirty-six patients were evaluable for PSA response to the first course of enzalutamide $( \pm$ PROSTVAC). The median PSA decline was $>99 \%$ (range $84->99 \%$ ) (figure $2 \mathrm{~A}$ ). Although median time to first PSA rise after completing 84 days of enzalutamide was 28 days, the range was broad (14-182 days) and the median time until PSA recovery to respective baseline value was 224 days (84-1246) after the shortcourse of therapy (figure $2 \mathrm{~B}$ ).

An amendment allowed for retreatment with 84 days of enzalutamide (without vaccine) and for those 26 patients the median PSA decline was again $>99 \% \quad(87->99)$ with a median time to recovery to second baseline PSA of 189 days (78-400) (figure 2). (Three patients on second course of therapy discontinued for toxicity (see below) and one discontinued due to travel logistics.) Only two patients on study developed metastatic disease; one was 451 days after starting treatment on cycle 1 and another was treated with two courses and had metastasis 574 days after initiating therapy.

\section{Toxicity}

The treatment of enzalutamide in the absence of ADT was well tolerated with a grade 3 event (elevated alanine aminotransferase/aspartate aminotransferase) seen in two patients one of whom was taking a prohibited herbal supplement (table 2). Resolution of most toxicities was rapid within weeks of discontinuing enzalutamide in 
A
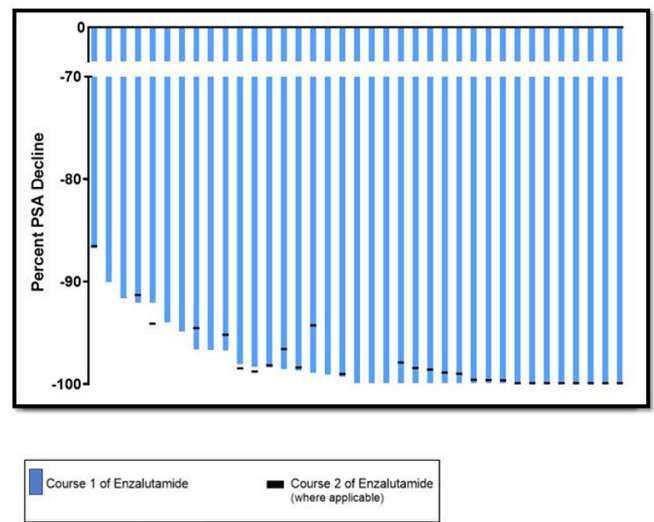

B

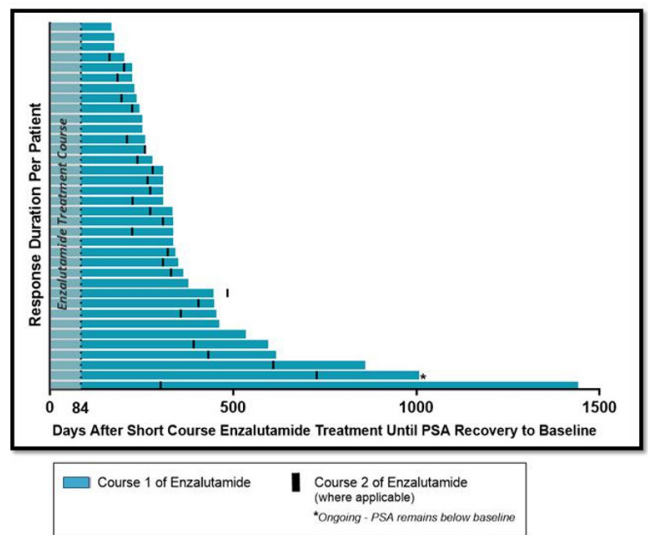

Figure 2 (A) This waterfall plot depicts maximum PSA response for each individual patient who completed the full 84-day initial course of enzalutamide. For the 28 patients who were treated with a second course of enzalutamide when PSA recovered to baseline after course 1 (including 2 patients who discontinued for toxicity) a paired bar is included. (B) The swimmers plot depicts the duration of PSA control below baseline for each individual patient including the 84-day course of enzalutamide (median $=224$ days after treatment, 308 days including treatment). For the 26 patients who completed a second course of enzalutamide, a paired bar is included (median = 189 days after treatment, 273 days including treatment).

cycle 1 . Vaccine-related toxicity (injection site reactions and flu-like symptoms) was seen frequently in the PROSTVAC cohort as expected. Fatigue was the most frequent adverse event seen ( $89 \%$ of all patients), but mostly grade 1 and transient with only seven patients (18\%) having grade 2 fatigue at any time point, regardless of vaccine. Breast tenderness was seen in most patients $(82 \%)$ but was universally grade 1 and likely in relation to elevation in systemic levels of testosterone. The second course of enzalutamide was stopped for precautionary reasons for dizziness and mild cognitive issues, in two patients respectively.

\section{Testosterone}

Testosterone increased in 22 of 36 patients beyond the institutional normal range, with a median rise to $834 \mathrm{ng}$ / dL (range 308-1579) with course 1 of enzalutamide and $721 \mathrm{ng} / \mathrm{dL}$ (227-1171) in course 2. Testosterone declined from peak values in patients on enzalutamide within 4-6 weeks of completing the 84-day course of therapy.

Table 2 Adverse events

\begin{tabular}{|c|c|c|c|c|c|c|c|c|c|}
\hline \multirow[b]{2}{*}{ Adverse Event } & \multicolumn{3}{|c|}{ Enzalutamide+Prostvac $(n=20)$} & \multicolumn{3}{|c|}{ Enzalutamide alone $(n=18)$} & \multicolumn{3}{|c|}{ Total for all patients $(n=38)$} \\
\hline & Grade 1 & Grade 2 & Grade 3 & Grade 1 & Grade 2 & Grade 3 & Grade1 & Grade 2 & Grade 3 \\
\hline Injection Site Reaction & $14(70 \%)$ & $4(20 \%)$ & 0 & 0 & 0 & 0 & $14(37 \%)$ & $4(11 \%)$ & 0 \\
\hline Fatigue/Lethargy & $14(70 \%)$ & $4(20 \%)$ & 0 & $13(72 \%)$ & $3(17 \%)$ & 0 & $27(71 \%)$ & $7(18 \%)$ & 0 \\
\hline $\begin{array}{l}\text { Breast Pain/Nipple } \\
\text { Tenderness }\end{array}$ & 17 (85\%) & 0 & 0 & 14 (78\%) & 0 & 0 & 31 (81\%) & 0 & 0 \\
\hline Myalgia & $4(20 \%)$ & 0 & 0 & $1(6 \%)$ & 0 & 0 & $5(13 \%)$ & 0 & 0 \\
\hline AST/ALT Elevation & 0 & 0 & $1(5 \%)$ & $3(17 \%)$ & 0 & $1(6 \%)$ & $3(8 \%)$ & 0 & $2(5 \%)$ \\
\hline Anorexia & $5(20 \%)$ & 0 & 0 & $2(11 \%)$ & 0 & & 7 (39\%) & 0 & 0 \\
\hline Dizziness & $3(15 \%)$ & $1(5 \%)$ & 0 & $2(11 \%)$ & $2(11 \%)$ & 0 & $5(28 \%)$ & $3(8 \%)$ & 0 \\
\hline Memory Impairment & $3(15 \%)$ & 0 & 0 & $1(6 \%)$ & 0 & 0 & $4(11 \%)$ & 0 & 0 \\
\hline Anemia & $6(30 \%)$ & 0 & 0 & $4(22 \%)$ & 0 & 0 & $10(26 \%)$ & 0 & 0 \\
\hline $\begin{array}{l}\text { Decreased White Blood } \\
\text { Cells }\end{array}$ & $5(25 \%)$ & $2(10 \%)$ & 0 & $3(17 \%)$ & 0 & 0 & 8 (31\%) & $2(5 \%)$ & 0 \\
\hline Decreased Libido & $3(15 \%)$ & 0 & 0 & $1(6 \%)$ & 0 & 0 & $4(22 \%)$ & 0 & 0 \\
\hline
\end{tabular}


Antigen-specific T cell responses, peripheral immune subsets, and thymic activity

Antigen-specific $\mathrm{T}$ cells targeting PSA were expanded after 3 months of therapy in a similar frequency of patients treated with enzalutamide alone $(7 / 15,45 \%)$ versus enzalutamide + vaccine $(6 / 15,40 \%)$. Similar findings were observed after 7 months of treatment, with $6 / 15(40 \%)$ patients receiving enzalutamide alone and $8 / 15(53 \%)$ patients receiving enzalutamide + vaccine displaying increases in PSA-specific T cells.

\section{Enzalutamide Alone}

A

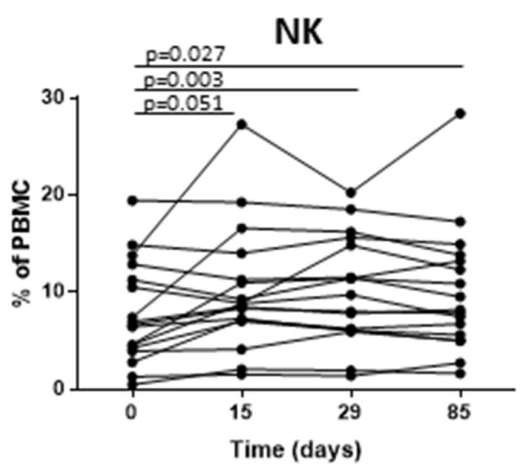

C

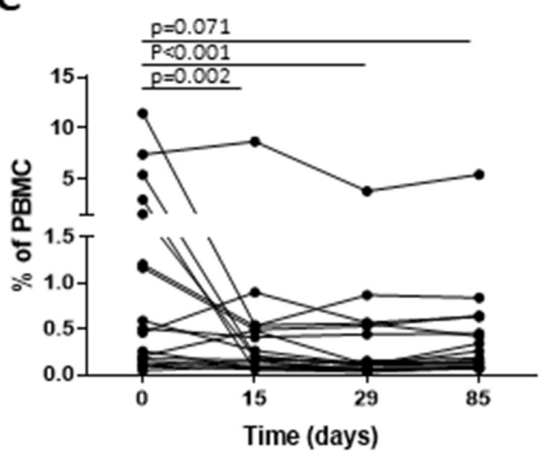

$\mathbf{E}$

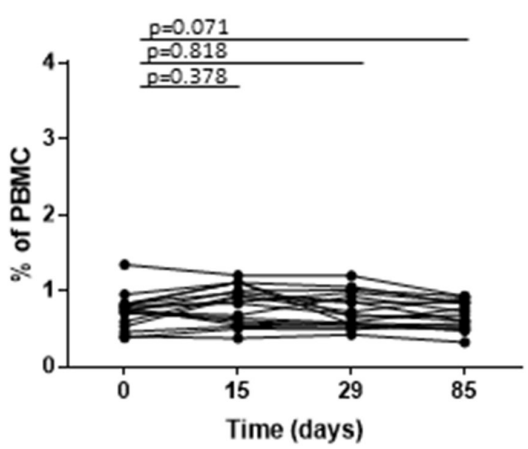

Analyses of PBMC subsets in patients treated with enzalutamide alone and in combination with vaccine revealed mainly immune potentiating effects (online supplemental table 1). Patients treated with enzalutamide alone had increases in NK cells (figure 3A), including mature NK cells and Tim3+ NK cells, as well as reductions in the immune suppressive MDSC compartment, including granulocytic MDSC (gMDSC, figure 3C) and programmed death ligand 1 (PD-L1+) MDSCs. Similar increases in the NK compartment were observed

\section{Enzalutamide + Vaccine}

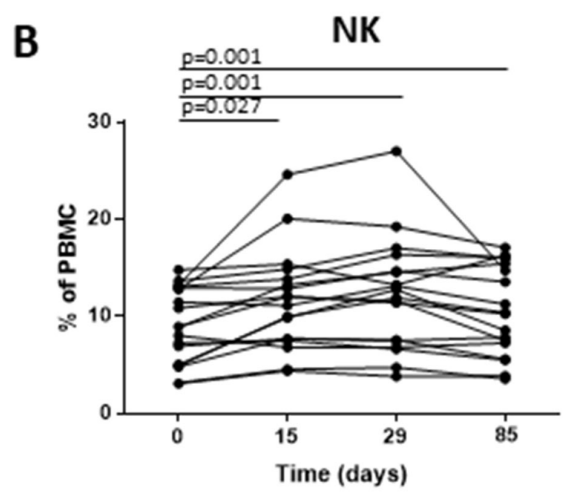

D

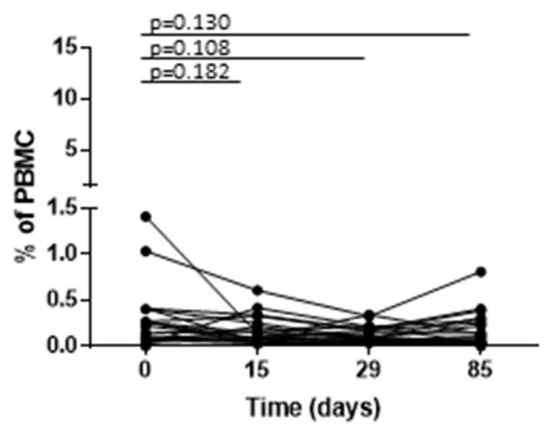

$\mathbf{F}$
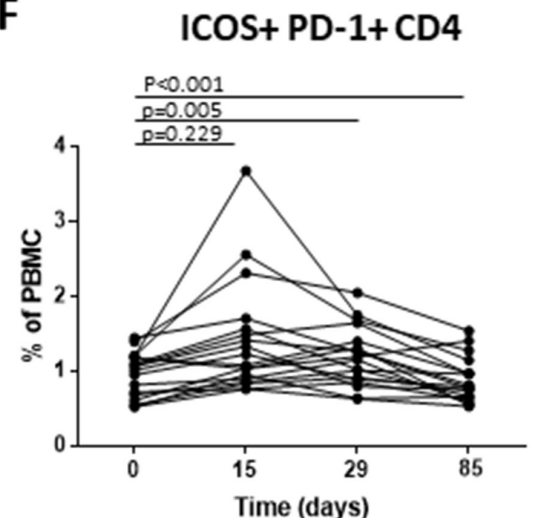

Figure 3 Frequency of notable PBMC subsets changing during enzalutamide +/-vaccine therapy. Increase in natural killer cells in patients treated with enzalutamide alone (A) or enzalutamide + vaccine (B). Decrease in granulocytic myeloid derived suppressor cells (gMDSC) in patients treated with enzalutamide alone (C), but not in patients treated with enzalutamide + vaccine (D). Increase in ICOS+PD1+ CD4 T cells in patients treated with enzalutamide + vaccine (F), but not in patients treated with enzalutamide alone (E). Subsets with a potentially biologically relevant change are were defined as those with $p<0.05$, the majority of patients having a $>25 \%$ change, difference in medians $>0.05 \%$ of PBMCs, and a frequency $>0.1 \%$ of total PBMCs. (Wilcoxon signed ranked test was used in this analysis). 


\section{D15}

vs Pre

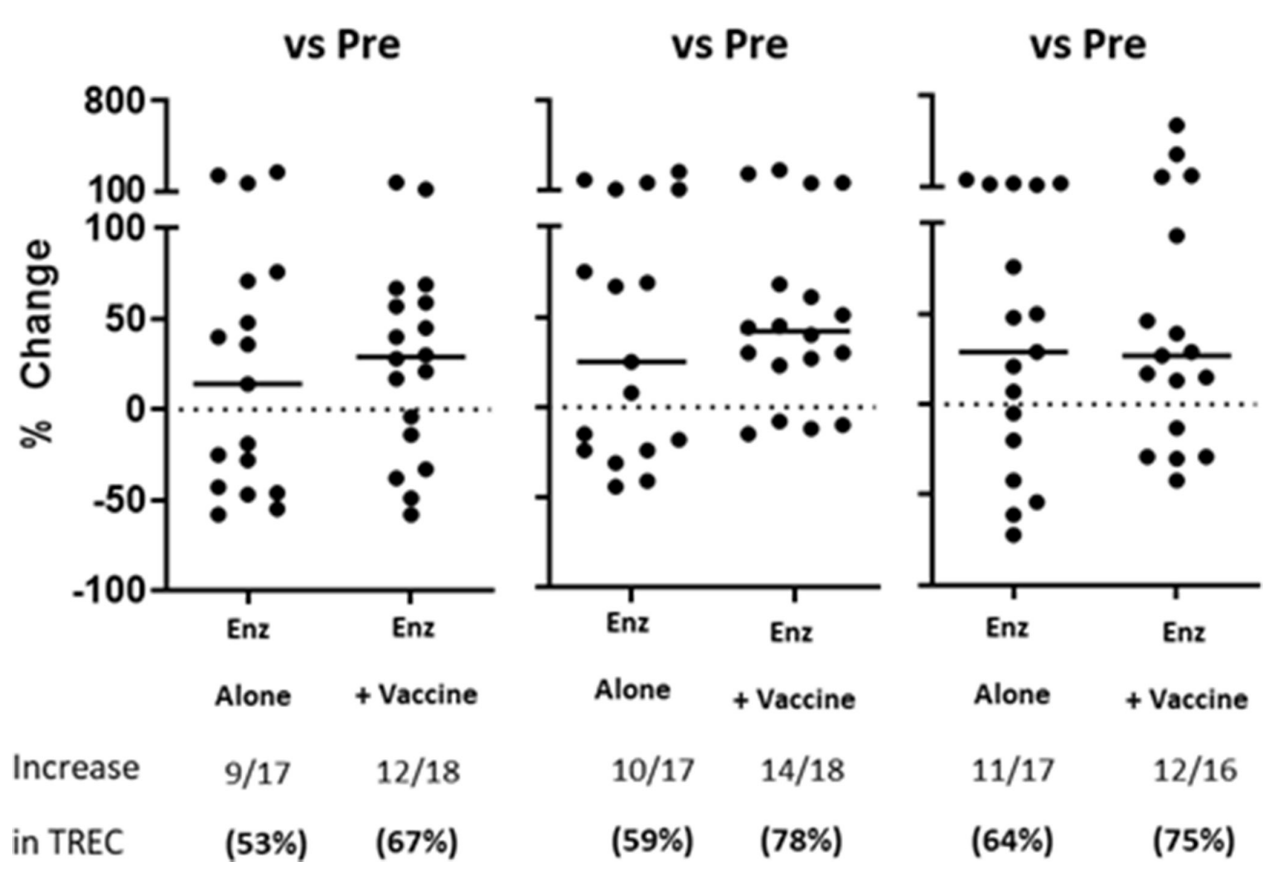

D29

D85

Figure 4 Percent change in TREC after Therapy. Data in graphs indicates the \% change in TREC/TRAC levels at the indicated time points after vs before therapy. Frequency and percentage of patients with increase in TREC are indicated. Bars indicate mean +/- SEM. DNA (100 ng) isolated from PBMCs was amplified to detect TREC and the housekeeper gene TRAC (T cell receptor alpha constant gene) using primer and probe sequences that have been previously described. ${ }^{19}$ Mean $\mathrm{Ct}$ values for the target gene (TREC) were normalized to mean Ct values for the housekeeping gene (TRAC) $[-\triangle \mathrm{Ct}=\mathrm{Ct}(\mathrm{TRAC})-\mathrm{Ct}(\mathrm{TREC})]$. The ratio of expression of the target gene TRAC versus TREC was defined as $2(-\triangle \mathrm{Ct}) .{ }^{20}$

in patients treated with enzalutamide plus vaccine (figure 3B); however, here there were no reductions in the MDSC compartment (figure 3D), and instead were increased in activated CD4 that coexpress inducible T cell costimulator (ICOS) and programmed cell death protein 1 (PD-1) (figure 3F). Although activated CD4 were increased only in the combination arm (Figure 3E,F), it is unclear whether these cells are antigen and/or vaccine specific. Representative flow plots of notable subsets that change with therapy are shown in online supplemental figure 3 .

TRECs were increased in the majority of evaluated patients (9 of 12) while on treatment with enzalutamide at time points between 15 and 86 days after initiation; TRECs were similarly increased in patients treated with enzalutamide combined with vaccine (figure 4). Furthermore, age was not a determining factor in the magnitude of increase in TRECs $(r=0.21 ; p=0.49)$. There was a declining trend of decreasing mean TREC levels noted within 14 days of stopping enzalutamide $(p=0.064)$.

\section{Serum VEGF}

There was no significant difference in the VEGF concentrations between those patients whoreceived PROSTVAC and those who received enzalutamide monotherapy. Eleven patients were evaluated during both courses of enzalutamide, while five were evaluated only in course 1 and six were evaluated only in course 2 . The mean pretreatment plasma VEGF concentration was $192 \mathrm{pg} /$
$\mathrm{mL}$, SD of $82 \mathrm{pg} / \mathrm{mL}$, median $174 \mathrm{pg} / \mathrm{mL}$. VEGF concentrations were compared over the course of treatment using paired, Wilcoxon signed rank test, and no significant difference was seen in plasma VEGF concentration over the course of treatment (online supplemental figure 4).

\section{DISCUSSION}

Since this trial was initiated, it is important to note that PROSTVAC alone failed to improve survival in a phase 3 trial in mCRPC. ${ }^{15}$ Although the use of PROSTVAC with enzalutamide did not improve PSA recovery rates, there was a more recent study of PROSTVAC alone in a similar nmCSPC population that suggested that PROSTVAC alone could induce delayed PSA declines in a subset of patients. ${ }^{31}$ Given the efficacy of enzalutamide to induce a median PSA decline of $99 \%$, it seems likely that any PSA signal of PROSTVAC efficacy was lost in that substantial response to enzalutamide, although it is noteworthy that the three patients with the greatest duration of PSA response below baseline in this study (for 861, 1008, and 1442 days, respectively) received vaccine with enzalutamide. Interestingly, there were increases in CD4 markers of activity (ICOS and PD-1) that were seen in the vaccine arm, but not the enzalutamide alone arm.

Despite the lack of an efficacy signal of enzalutamide and vaccine, this study is notable because it demonstrates 
for the first time that short-course enzalutamide (regardless of vaccine and without ADT) in nmCSPC brought about prolonged PSA control well beyond the treatment period and such responses were largely duplicated with a second course of enzalutamide for 3 months. The median duration of 224 days after 84 days of treatment until PSA recovery to baseline means that for men with nmCSPC and rising PSA, a 3 month course of enzalutamide afforded them PSA control (ie, PSA below baseline) for 308 days (11 months) and a second 3 month course in eligible patients afforded them another median 273 days (9.75 months) with a PSA below baseline. It is perhaps not surprising that some benefit was diminished in duration of the second course (although median PSA declines were $>99 \%$ in both groups). For the two patients who had metastatic recurrence at first and second PSA rise, it was only after an extended response of 451 and 574 days, respectively. These findings are noteworthy because it suggests that men with nmCSPC who may have been treated previously with continuous or intermittent ADT may now experience effective PSA control with intermittent short-course enzalutamide and less toxicity than ADT.

In addition to intermittent $\mathrm{ADT}$, there have been other short-course therapy strategies explored in men with nmCSPC but they appear to be either more toxic and/ or less effective. One study administered $80 \mathrm{mg}$ of bicalutamide monotherapy for 2 years in men postprostatectomy (with no minimum required PSA DT) ${ }^{32}$ Of 91 patients on the continuous regimen, $26.4 \%$ had progression within 2 years, but $33 \%$ remain without progression at 5 years. Perhaps this study in an unselected population further highlights the potential role of androgen receptor disruption as monotherapy for abbreviated courses. Higher doses of bicalutamide have been investigated in this population, but also had increased toxicity. ${ }^{33} \mathrm{~A}$ more recent strategy evaluated an 8 month course of abiraterone with ADT versus $\mathrm{ADT}$ alone in 197 men with nmCSPC. The results suggest that the addition of abiraterone to ADT improved PSA-free survival from a median of 21.1 months to 28.3 months, but the use of ADT increased the toxicity profile of this strategy. ${ }^{34}$

While some practitioners hold firm to the stance of not treating nmCSPC until the development of metastatic disease, there may be some benefits to the use of ADT in clinical practice. Some patients have anxiety about rising PSA levels and ADT, despite its side effects, may improve quality of life by decreasing anxiety along with PSA. (Our future studies in nmCSPC will look into quality of life impact prospectively). Another major concern is that not all men with nmCSPC develop metastasis. While that is an accurate statement, subsets of patients with rapid PSA DTs less than 3 months have a $50 \%$ chance of developing metastasis within 1 year. ${ }^{9}$ Such populations should be considered for more aggressive therapies to prevent metastasis and the morbidity associated with metastatic disease. Patients who develop metastasis will require ADT indefinitely and then must contend with the associated toxicities. If the use of intermittent short-course enzalutamide can control PSA for nearly a year, and then be repeated with minimal diminishing benefit and toxicity, it is possible that such PSA control would come with a delay in metastasis in men who have rapidly rising PSAs. Thus, short-course enzalutamide could potential defer the need for indefinite ADT (and associated toxicities) in these patients, although future trials would be required to confirm this hypothesis.

The previous study of enzalutamide in nmCSPC was associated with some degree of toxicity, which was similarly seen in this trial (with grade 1 fatigue and breast/ nipple pain predominating, and rare grade 3 toxicities). While these symptoms may appear minor, it is important to recall that nmCSPC patients have no cancer-related symptoms and thus these likely cumulative symptoms will impact quality of life over time and could diminish treatment compliance. Such toxicities are likely to be apparent in the ongoing EMBARK trial (NCT02319837), a phase 3 trial evaluating enzalutamide \pm ADT in nmCSPC. Much like intermittent ADT was associated with improved quality of life due to periods where patients were not on therapy, it is likely that intermittent enzalutamide could afford similar benefits, as this study demonstrates, while also being associated with prolonged PSA control. ${ }^{12}$

The immunologic impact of enzalutamide independent of vaccine in this study is important as well. To our knowledge, the immune effects of enzalutamide without ADT have not been previously reported. Enzalutamide was associated with increased subpopulations of activated NK cells and decreases in immune suppressive gMDSCs. Emerging data have suggested that within the prostate cancer immune microenvironment NK cells and MDSCs may be more relevant than $\mathrm{T}$ cells. ${ }^{35} 36$ The apparent minimal impact seen on $\mathrm{T}$ cells after enzalutamide alone could provide insight into the recent phase 3 trial that demonstrated no improvement when the PDL1 targeting antibody atezolizumab was added to enzalutamide in advanced prostate cancer. ${ }^{37}$

We also report for the first time in humans that an androgen receptor targeting therapy can increase naïve $\mathrm{T}$ cell production from the thymus in humans. TRECS are the byproduct of $\mathrm{T}$ cell receptor gene rearrangement that occurs when naive $\mathrm{T}$ cells are produced from the thymus, thereby providing an indirect measure of thymic activity. Previous studies have shown that androgen blockade in mice and humans activates thymic regeneration and increases TREC levels. ${ }^{38}$ Similar to androgen deprivation, 2 weeks of enzalutamide treatment in C57BL/ 6 mice increased thymic weight and TREC production. ${ }^{14}$ The increases in TRECs coincided with therapy, irrespective of age. Interestingly, even though the impact of enzalutamide on the PSA often lasts months after therapy, TREC levels appeared to decline 2 weeks after enzalutamide discontinuation.

There was no clear association between clinical responses and immune changes, but most patients in this study had sustained PSA declines of large magnitude so 
such associations may not be readily apparent in a trial of this size.

The immune findings in this study are remarkable because, beyond sipuleucel- $T$, immune checkpoint inhibitors are only effective in about half of patients who possess rare mutations like microsatellite instability and CDK12 inactivation. ${ }^{39} 40$ Since enzalutamide is already frequently used in many stages of prostate cancer, additional immunotherapies can be added to exploit the immune potentiating effects of enzalutamide on NK cells and gMDSCs. While PD1/PDL1 inhibitors are often dependent on T cells infiltrating the tumor for their effects, other immunotherapies such as immunocytokines could be combined with enzalutamide to bring about a synergistic clinical impact. The findings of this study highlight that the best opportunities may lie in focusing on NK cells when developing immune-based combinations with enzalutamide. Additional strategies could be built on enzalutamide's ability to enhance TRECs and possibly be used beyond prostate cancer.

Although the trial is small in numbers, the median PSA decline of $>99 \%$ is likely to be a reasonable indicator of the potential for short-course enzalutamide in nmCSPC. While the responses to the second course of enzalutamide are encouraging, it should be noted that some degree of selection bias may have impacted why some patients did not receive a second course, either by protocol requirement or other factors.

A shortcoming of this trial stems from the fact that it was initiated in 2013; thus it did not incorporate modern positron emission tomography (PET) or molecular imaging strategies, which are increasingly being used in clinical practice. It is worth noting that many patients with $\mathrm{nmCSPC}$ are likely to have micrometastatic disease detectable on sensitive PET imaging studies. ${ }^{41}$ Nonetheless, these findings on ultra-sensitive modern imaging studies do not meet the conventional definition of metastasis as they have been defined in MCSPC clinical trials to this point (that is findings on CT/MRI or Tc99 bone scan). While some practitioners may contend that PET scan findings define metastatic disease that should be treated with docetaxel or antiandrogen therapies based on studies of those agents in overtly metastatic disease (as seen on CT and Tc99 bone scan), prospective data supporting this are lacking in either mCSPC or mCRPC trials where modern imaging techniques were not employed. Others may suggest radiation has an emerging role in oligometastatic prostate cancer;however, its role in oligorecurrence (rising PSA with negative conventional imaging after definitive therapy) is less defined.

Future studies of agents such as enzalutamide in nmCSPC need to be done to better understand their clinical role in this minimal residual disease state of prostate cancer. The data presented here provide a rationale for intermittent use of enzalutamide in men with biochemically recurrent prostate cancer that can impact PSA and thus likely decrease near term risk of metastatic disease, without the systemic toxicity of ADT. In order to define the impact beyond just PSA, such studies should require baseline and follow-up assessments with modern (PET) imaging to better understand the antitumor effects that are associated with transient but substantial and repeatable PSA declines such as those seen with short-course enzalutamide in this study. If such responses are associated with changes in PET imaging (ie, improvements) and/or the delay in ultimate progression of disease in PET or conventional imaging, then perhaps such an intermediate endpoint could be evaluated in phase 3 trials, as has been suggested by data from the ICECaP Working Group, demonstrating a strong association between metastasisfree survival and overall survival in localized disease. ${ }^{42}$ Indeed, modern imaging has potentially lifted the shroud of nmCSPC, allowing for more pragmatic treatment of the minimal residual disease state in patients who are at high risk for metastasis.

Twitter Ravi A Madan @Dr_RaviMadan, Marijo Bilusic @mbilusic and James L Gulley @gulleyj1

Acknowledgements Editorial acknowledgement. Debra Weingarten, Laboratory of Tumor Immunology and Biology, Center for Cancer Research, National Cancer Institute.

Contributors RAM — conceptualization, methodology, validation, investigation, formal analysis, writing —original draft, visualization, supervision, project administration, funding acquisition. FK-investigation, writing — review and editing RD—methodology, investigation, formal analysis, writing —original draft. MA-Hformal analysis, writing — original draft. MB — investigation, writing — review and editing. ILR - investigation, writing — review and editing. HS—investigation, writing — review and editing. PMA—investigation, writing—review and editing. MRT—investigation, writing — review and editing. JLM —investigation, writing review and editing, formal analysis. LMC—investigation, writing—review and editing. AC—investigation, writing — review and editing. $\mathrm{AH}$ —investigation, writing — review and editing. MW—investigation, writing — review and editing. $\mathrm{NH}$-investigation, writing - review and editing, data curation. $\mathrm{HO}$-investigation, writing — review and editing, data curation. SEL-investigation, writing —review and editing. $\mathrm{CHC}$-investigation, writing — review and editing. SMS—investigation, writing — review and editing, conceptualization. WDF-investigation, writingreview and editing. JS—investigation, writing — review and editing. WLDinvestigation, writing — review and editing, conceptualization, resources. JLGinvestigation, writing — review and editing, conceptualization, resources.

Funding This study was funded by the Intramural Program at the National Cancer Institute, Bethesda, Maryland, USA. It was also supported with drug supply from Astellas Pharma Global Development/Pfizer and Bavarian Nordic.

Competing interests No, there are no competing interests.

Patient consent for publication Not required.

Ethics approval All patients signed Institutional Review Board-approved informed consents.

Provenance and peer review Not commissioned; externally peer reviewed.

Data availability statement All data relevant to the study are included in the article or uploaded as supplementary information. All data relevant to the study are included in the article or uploaded as supplementary information.

Supplemental material This content has been supplied by the author(s). It has not been vetted by BMJ Publishing Group Limited (BMJ) and may not have been peer-reviewed. Any opinions or recommendations discussed are solely those of the author(s) and are not endorsed by BMJ. BMJ disclaims all liability and responsibility arising from any reliance placed on the content. Where the content includes any translated material, BMJ does not warrant the accuracy and reliability of the translations (including but not limited to local regulations, clinical guidelines, terminology, drug names and drug dosages), and is not responsible for any error and/or omissions arising from translation and adaptation or otherwise.

Open access This is an open access article distributed in accordance with the Creative Commons Attribution Non Commercial (CC BY-NC 4.0) license, which 
permits others to distribute, remix, adapt, build upon this work non-commercially, and license their derivative works on different terms, provided the original work is properly cited, appropriate credit is given, any changes made indicated, and the use is non-commercial. See http://creativecommons.org/licenses/by-nc/4.0/.

\section{ORCID iDs}

Ravi A Madan http://orcid.org/0000-0001-5106-8636

Marijo Bilusic http://orcid.org/0000-0003-1020-689X

Lisa Cordes http://orcid.org/0000-0003-3833-4084

Jeffrey Schlom http://orcid.org/0000-0001-7932-4072

James L Gulley http://orcid.org/0000-0002-6569-2912

\section{REFERENCES}

1 Beer TM, Armstrong AJ, Rathkopf DE, et al. Enzalutamide in metastatic prostate cancer before chemotherapy. N Engl J Med 2014;371:424-33.

2 Scher HI, Fizazi K, Saad F, et al. Increased survival with enzalutamide in prostate cancer after chemotherapy. $N$ Engl J Med 2012;367:1187-97

3 Davis ID, Martin AJ, Stockler MR, et al. Enzalutamide with standard first-line therapy in metastatic prostate cancer. $N$ Engl J Med 2019;381:121-31.

4 Hussain M, Fizazi K, Saad F, et al. Enzalutamide in men with nonmetastatic, castration-resistant prostate cancer. N Engl J Med 2018;378:2465-74.

5 Spratt DE, McHugh DJ, Morris MJ, et al. Management of biochemically recurrent prostate cancer: ensuring the right treatment of the right patient at the right time. Am Soc Clin Oncol Educ Book 2018;38:355-62.

6 Loblaw A, Bassett J, D'Este C, et al. Timing of androgen deprivation therapy for prostate cancer patients after radiation: planned combined analysis of two randomized phase 3 trials. J Clin Oncol 2018;36:5018.

7 Paller CJ, Antonarakis ES. Management of biochemically recurrent prostate cancer after local therapy: evolving standards of care and new directions. Clin Adv Hematol Oncol 2013;11:14-23.

8 Markowski MC, Chen Y, Feng Z, et al. Psa doubling time and absolute PSA predict metastasis-free survival in men with biochemically recurrent prostate cancer after radical prostatectomy. Clin Genitourin Cancer 2019;17:470-5.

9 Antonarakis ES, Feng Z, Trock BJ, et al. The natural history of metastatic progression in men with prostate-specific antigen recurrence after radical prostatectomy: long-term follow-up. BJU Int 2012;109:32-9.

10 Suzman DL, Zhou XC, Zahurak ML, et al. Change in PSA velocity is a predictor of overall survival in men with biochemically-recurrent prostate cancer treated with nonhormonal agents: combined analysis of four phase-2 trials. Prostate Cancer Prostatic Dis 2015;18:49-55.

11 Tombal B, Borre M, Rathenborg P, et al. Enzalutamide monotherapy in hormone-naive prostate cancer: primary analysis of an open-label, single-arm, phase 2 study. Lancet Oncol 2014;15:592-600.

12 Crook JM, O'Callaghan CJ, Duncan G, et al. Intermittent androgen suppression for rising PSA level after radiotherapy. $N$ Engl J Med 2012;367:895-903.

13 Singh P, Pal SK, Alex A, et al. Development of PROSTVAC immunotherapy in prostate cancer. Future Oncol 2015;11:2137-48.

14 Ardiani A, Farsaci B, Rogers CJ, et al. Combination therapy with a second-generation androgen receptor antagonist and a metastasis vaccine improves survival in a spontaneous prostate cancer model. Clin Cancer Res 2013;19:6205-18.

15 Gulley JL, Borre M, Vogelzang NJ, et al. Phase III trial of PROSTVAC in asymptomatic or minimally symptomatic metastatic castrationresistant prostate cancer. J Clin Oncol 2019;37:1051-61.

16 Stein WD, Gulley JL, Schlom J, et al. Tumor regression and growth rates determined in five intramural $\mathrm{NCl}$ prostate cancer trials: the growth rate constant as an indicator of therapeutic efficacy. Clin Cancer Res 2011:17:907-17.

17 Gatti-Mays ME, Strauss J, Donahue RN, et al. A phase I doseescalation trial of BN-CV301, a recombinant Poxviral vaccine targeting MUC1 and CEA with costimulatory molecules. Clin Cancer Res 2019;25:4933-44.

18 Terasawa $\mathrm{H}$, Tsang $\mathrm{K}-\mathrm{Y}$, Gulley J, et al. Identification and characterization of a human agonist cytotoxic T-lymphocyte epitope of human prostate-specific antigen. Clin Cancer Res 2002;8:41-53.

19 Donahue RN, Lepone LM, Grenga I, et al. Analyses of the peripheral immunome following multiple administrations of avelumab, a human IgG1 anti-PD-L1 monoclonal antibody. J Immunother Cancer 2017;5:20.
20 Lepone LM, Donahue RN, Grenga I, et al. Analyses of 123 peripheral human immune cell subsets: defining differences with age and between healthy donors and cancer patients not detected in analysis of standard immune cell types. J Circ Biomark 2016;5:5.

21 Strauss J, Heery CR, Schlom J, et al. Phase I trial of M7824 (MSB0011359C), a bifunctional fusion protein targeting PD-L1 and TGF $\beta$, in advanced solid tumors. Clin Cancer Res 2018;24:1287-95.

22 Rajan A, Heery CR, Thomas A, et al. Efficacy and tolerability of anti-programmed death-ligand 1 (PD-L1) antibody (Avelumab) treatment in advanced thymoma. J Immunother Cancer 2019;7:269.

23 Bilusic M, Heery CR, Collins JM, et al. Phase I trial of HuMax-IL8 (BMS-986253), an anti-IL-8 monoclonal antibody, in patients with metastatic or unresectable solid tumors. J Immunother Cancer 2019;7:240.

24 Strauss J, Heery CR, Kim JW, et al. First-In-Human phase I trial of a tumor-targeted cytokine (NHS-IL12) in subjects with metastatic solid tumors. Clin Cancer Res 2019;25:99-109.

25 Mensen A, Ochs C, Stroux A, et al. Utilization of TREC and KREC quantification for the monitoring of early $\mathrm{T}$ - and $\mathrm{B}$-cell neogenesis in adult patients after allogeneic hematopoietic stem cell transplantation. J Transl Med 2013;11:188.

26 Livak KJ, Schmittgen TD. Analysis of relative gene expression data using real-time quantitative PCR and the 2(-Delta Delta C(T)) Method. Methods 2001;25:402-8.

27 Fernandez EV, Reece KM, Ley AM, et al. Dual targeting of the androgen receptor and hypoxia-inducible factor $1 \alpha$ pathways synergistically inhibits castration-resistant prostate cancer cells. Mol Pharmacol 2015;87:1006-12.

28 Luo Y, Azad AK, Karanika S, et al. Enzalutamide and CXCR7 inhibitor combination treatment suppresses cell growth and angiogenic signaling in castration-resistant prostate cancer models. Int $J$ Cancer 2018;142:2163-74

29 Madan RA, Bilusic M, Heery C, et al. Clinical evaluation of TRICOM vector therapeutic cancer vaccines. Semin Oncol 2012;39:296-304.

30 DiPaola RS, Chen Y-H, Bubley GJ, et al. A national multicenter phase 2 study of prostate-specific antigen (PSA) pox virus vaccine with sequential androgen ablation therapy in patients with PSA progression: ECoG 9802. Eur Urol 2015;68:365-71.

31 Madan RA, Karzai F, Bilusic M, et al. Immunotherapy for biochemically recurrent prostate cancer. J Clin Oncol 2018;36:215.

32 Okubo T, Mitsuzuka K, Koie T, et al. Two years of Bicalutamide monotherapy in patients with biochemical relapse after radical prostatectomy. Jpn J Clin Oncol 2018;48:570-5.

33 See WA, Wirth MP, McLeod DG, et al. Bicalutamide as immediate therapy either alone or as adjuvant to standard care of patients with localized or locally advanced prostate cancer: first analysis of the early prostate cancer program. J Urol 2002;168:429-35.

34 Efstathiou E, Wang X, Amado JZ. A randomized study of finite abiraterone acetate (AA) plus leuprolide ( $\mathrm{LHRHa}$ ) versus LHRHa in biochemically recurrent non metastatic hormone naïve prostate cancer (MOHNPC). J Clin Oncol 2018;35:5002.

35 Zhao SG, Lehrer J, Chang SL, et al. The immune landscape of prostate cancer and nomination of PD-L2 as a potential therapeutic target. JNCl: Journal of the National Cancer Institute 2019;111:301-10.

36 Calcinotto A, Spataro C, Zagato E, et al. II-23 secreted by myeloid cells drives castration-resistant prostate cancer. Nature 2018;559:363-9.

37 Sweeny CJ, Gillessen S, Rathkopf D, et al. Abstract CT014, IMbassador250: a phase III trial comparing atezolizumab with enzalutamide vs enzalutamide alone in patients with metastatic castration-resistant prostate cancer (mCRPC). American Association for Cancer Research (AACR) Virtual Annual Meeting I; April 27-28 2020.

38 Sutherland JS, Goldberg GL, Hammett MV, et al. Activation of thymic regeneration in mice and humans following androgen blockade. $J$ Immunol 2005;175:2741-53.

39 Graham LS, Montgomery B, Cheng HH, et al. Mismatch repair deficiency in metastatic prostate cancer: response to PD-1 blockade and standard therapies. PLoS One 2020;15:e0233260.

40 Reimers MA, Yip SM, Zhang L, et al. Clinical outcomes in cyclindependent kinase 12 mutant advanced prostate cancer. Eur Urol 2020;77:333-41.

41 Eiber M, Rauscher I, Souvatzoglou M, et al. Prospective head-tohead comparison of ${ }^{11} \mathrm{C}$-choline-PET/MR and ${ }^{11} \mathrm{C}$-choline-PET/CT for restaging of biochemical recurrent prostate cancer. Eur J Nucl Med Mol Imaging 2017;44:2179-88.

42 Xie W, Regan MM, Buyse M, et al. Metastasis-Free survival is a strong surrogate of overall survival in localized prostate cancer. J Clin Oncol 2017;35:3097-104. 